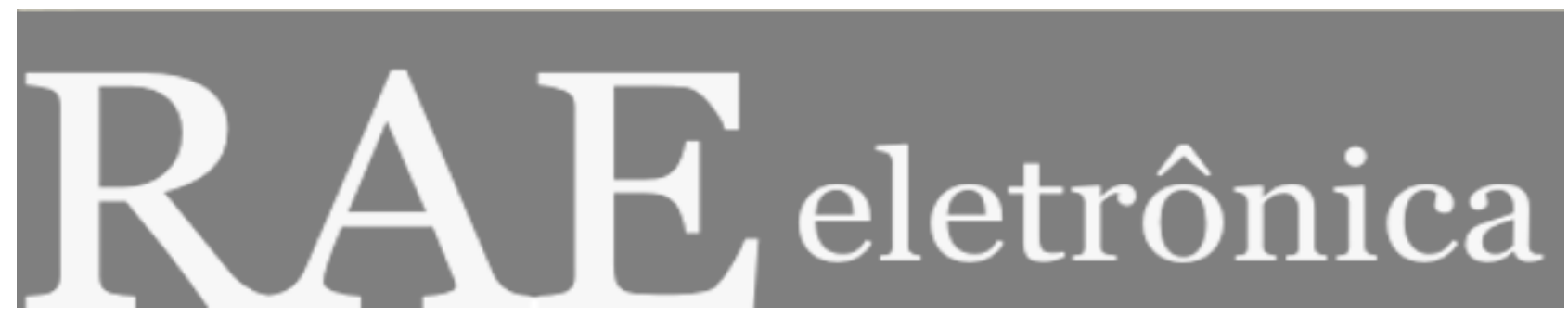

\title{
USO DE FONTES DE RECOMENDAÇÃO FORTES E FRACAS NA ESCOLHA DE VEÍCULO
}

Por:

Mateus Canniatti Ponchio

Francisco José Epósito Aranha Filho

André Luiz Silva Samartini

RAE-eletrônica, Volume 2, Número 1, jan-jun/2003.

http://www.rae.com.br/eletronica/index.cfm?FuseAction=Artigo\&ID=1267\&Secao=MERCADO\&Volume=2\&Numero $=1 \&$ Ano $=2003$

CCopyright, 2002, RAE-eletrônica. Todos os direitos, inclusive de tradução, são reservados. É permitido citar parte de artigos sem autorização prévia desde que seja identificada a fonte. A reprodução total de artigos é proibida. Os artigos só devem ser usados para uso pessoal e não-comercial. Em caso de dúvidas, consulte a redação: redacao@rae.com.br.

A RAE-eletrônica é a revista on-line da FGV-EAESP, totalmente aberta e criada com o objetivo de agilizar a veiculação de trabalhos inéditos. Lançada em janeiro de 2002, com perfil acadêmico, é dedicada a professores, pesquisadores e estudantes. Para mais informações consulte o site www.rae.com.br/eletronica.

RAE-eletrônica

ISSN 1676-5648

(C)2002 Editora: Fundação Getulio Vargas - Escola de Administração

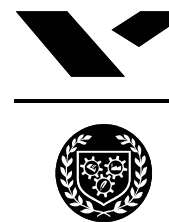

F U N D A C A A 0 GETULIO VARGAS

de Empresas de São Paulo. 


\title{
USO DE FONTES DE RECOMENDAÇÃO FORTES E FRACAS NA ESCOLHA DE VEÍCULO
}

\author{
Mateus Canniatti Ponchio \\ Mestrando em Administração de Empresas - FGV-EAESP \\ E-mail: mateuscp@gvmail.br
}

Endereço: R. Ab́́lio Soares, 438 apto 81 São Paulo - SP, 04005-001

Interesses de pesquisa: Comportamento do Consumidor e Marketing Quantitativo.

\section{Francisco José Epósito Aranha Filho}

Professor do Departamento de Informática e de Métodos Quantitativos Aplicados à Administração da FGV-EAESP e Consultor em Marketing de Precisão.

E-mail: francisco.aranha@,fgvsp.br

Endereço: Av. Nove de Julho, 2029, 11 o andar, IMQ São Paulo - SP, 01313-902

Interesses de pesquisa: Marketing de Precisão, Data Mining e Escalas de Medida.

\section{André Luiz Silva Samartini}

Professor do Departamento de Informática e de Métodos Quantitativos Aplicados à Administração da FGV-EAESP e Consultor em Marketing de Precisão.

E-mail: andre@fgvsp.br

Endereço: Av. Nove de Julho, 2029, 11 o andar, IMQ São Paulo - SP, 01313-902

Interesses de pesquisa: Métodos Quantitativos Aplicados ao Marketing

\section{RESUMO}

DUHAN et al. (1997) propuseram um modelo de nove caminhos causais para a intensidade de uso de fontes fortes e fracas na etapa de coleta de informações do processo de compra, apresentando evidência estatística para oito deles. PIRES e MARCHETTI (2000) aplicaram o modelo de DUHAN et al. na análise dos fatores que influenciam a escolha da fonte de informação na compra de automóveis novos, encontrando evidência para apenas quatro dos caminhos originalmente propostos. No presente artigo, descreve-se um experimento implementado por computador e disponibilizado pela Internet, com nível de dificuldade da tarefa controlado, em que se simula a escolha de automóveis novos. Os resultados obtidos fornecem evidência para também quatro das relações propostas por DUHAN et al. Tomadas em conjunto, as três pesquisas indicam fragilidade nas relações postuladas entre "conhecimento prévio" e as variáveis de ligação ("dificuldade da tarefa" e "importância de informações instrumentais e afetivas"). Sugere-se que os caminhos comprovados apenas por DUHAN poderiam ser verificados nas duas pesquisas seguintes mediante segmentação do perfil comportamental dos consumidores, hipótese oferecida como possibilidade para novas pesquisas.

\section{ABSTRACT}

DUHAN et al. (1997) proposed a model of nine causal paths for the intensity of use of strong and weak recommendation sources during the information collection stage of the purchase process, submitting statistical evidence for eight of those. PIRES and MARCHETTI (2000) applied the DUHAN et al. model to the analysis of factors that have a bearing on the choice of the source of information as regards the purchase of new cars, finding evidence of only four of the originally proposed paths. This article describes a computer-based experiment made available through the Internet, with controlled task difficulty level, in which the choice of new cars is simulated. The results obtained also provide evidence of four of the relationships proposed by DUHAN et al. Taken collectively, the three studies indicate the frailty of the relationships postulated between "prior knowledge" and the connection variables ("task difficulty" and the "importance of instrumental 
and affective information"). It is suggested that the paths proved by DUHAN alone might be verified in the two latter studies by segmenting the consumers' behavioral profile, a hypothesis that arises as a possibility for future research.

PALAVRAS-CHAVE

Automóveis - Coleta de Informação - Fontes de Informação - Internet - Processo de Decisão de Compra

\section{KEY-WORDS}

Automobiles - Gathering of Information - Recommendation Sources - Internet - Purchase Process 


\section{INTRODUÇÃO}

\section{Coleta de Informação e Fontes de Recomendações}

A escolha de um produto para consumo envolve uma seqüência típica de eventos, chamada de processo de compra: reconhecimento do problema, busca de informações, avaliação de alternativas, decisão de compra e comportamento pós-compra. O número de etapas percorridas e o tempo consumido em cada etapa variam entre indivíduos, produtos e circunstâncias de compra (NEWMAN e STAELIN, 1972 e 1973; PUNJ e STAELIN, 1983; PUTSIS JR. e SRINIVASAN, 1994; KOTLER, 1998). Este artigo focaliza a etapa de busca de informações.

Antes de comprar, o consumidor típico envolve-se num processo de coleta de informações limitado e seletivo, apoiando sua decisão final em uma fração pequena do total de informações disponíveis (NEWMAN e STAELIN, 1972; MIDGLEY, 1983; BEATTY e SMITH, 1987).

Para reduzir o montante de informação a ser processada, o consumidor adota estratégias simplificadoras em que troca certeza de realizar uma decisão correta por eficiência no processo decisório. Entre estas estratégias destaca-se a obtenção de recomendações de várias fontes. As recomendações ajudam a restringir o número de alternativas a considerar e o número de atributos avaliados em cada alternativa (OLSHAVSKY e GRANBOIS, 1979; DUHAN et al, 1997). Uma questão de interesse teórico (e de interesse prático no delineamento de programas de comunicação) é, portanto, identificar os fatores que influenciam a forma como o consumidor se serve das fontes de recomendação disponíveis (MIDGLEY, 1983; DUHAN et al, 1997).

\section{O Modelo de Duhan et al.}

No modelo apresentado por DUHAN et al. (1997) e utilizado como quadro de referência neste artigo, as fontes de recomendações são classificadas em fortes e fracas conforme conheçam o consumidor pessoalmente ou não. Exemplos de fontes fortes são os familiares, amigos e colegas de trabalho do consumidor; exemplos de fontes fracas são bibliotecas, meios de comunicação de massa, especialistas e agências governamentais. A vantagem oferecida pelas fontes fortes é o conhecimento simultâneo do produto e do consumidor, o que permite à fonte fazer recomendações personalizadas; a vantagem oferecida pelas fontes fracas é um conhecimento mais técnico e especializado sobre o produto.

$\begin{array}{lcc}\text { Grau de conhecimento } & \text { Variáveis de } & \text { Tipo de fonte de } \\ \text { prévio do consumidor } & \text { ligação } & \text { informação }\end{array}$

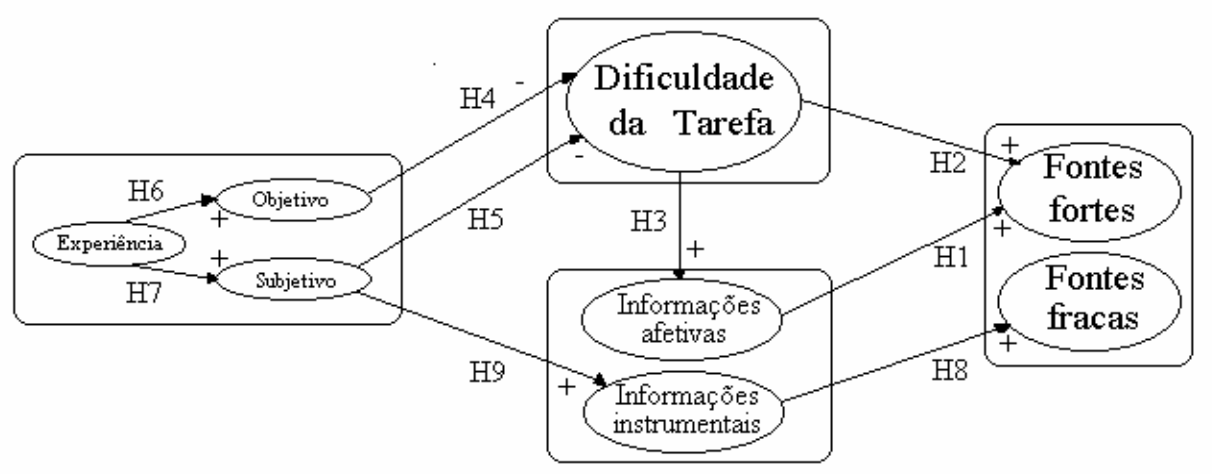

Figura 1 - Modelo Teórico da Escolha de Fonte de Recomendações (DUHAN et al, 1997) 
A Figura 1 apresenta as oito variáveis integrantes do modelo. Influenciando o número de fontes fortes e fontes fracas consultadas pelo consumidor há:

O tipo de informações consideradas importantes. Nesta dimensão, as informações podem ser instrumentais, isto é, relacionadas a aspectos técnicos ou de desempenho dos produtos e geralmente avaliadas independentemente do consumidor - por exemplo, no juízo: "este carro roda muitos quilômetros por litro de combustível" (BEN-SIRA, 1976 e 1980); ou afetivas, isto é, relacionadas a aspectos estéticos e geralmente avaliadas em relação ao consumidor - por exemplo, no juízo: "minha mulher odeia carros azuis" (BEN-SIRA, 1976 e 1980).

O grau de dificuldade percebido na decisão de compra. Este construto reflete o número de alternativas consideradas, o número de atributos avaliados em cada alternativa, e a simplicidade, abstração e redundância da linguagem na qual as informações são apresentadas (PAQUETTE e KIDA, 1988; KIM e KHOURY, 1987; PAYNE, 1976; NEWELL e SIMON, 1972).

O nível de conhecimento anterior sobre o produto. Este construto indica o grau de experiência e familiaridade do consumidor em relação ao produto e é medido em três componentes: o conhecimento subjetivo reflete a percepção que o consumidor tem de seu conhecimento (BRUCKS, 1985); o conhecimento objetivo baseia-se no conteúdo e na organização do conhecimento disponível na memória do consumidor (BRUCKS, 1997); e a experiência quantifica o conhecimento acumulado no uso direto do produto (PUNJ e STAELIN, 1983 e BETTMAN e PARK, 1980).

Interligando as oito variáveis do modelo, DUHAN et al. postulam, na forma de hipóteses, nove relações causais:

Hipótese 1: "Quanto maior a importância das informações afetivas, mais provável a busca de recomendações de fonte forte";

Hipótese 2: "Quanto maior o nível de dificuldade percebido na escolha, mais provável a busca de recomendações de fonte forte";

Hipótese 3: "Quanto maior o nível de dificuldade percebido na escolha, maior a importância de informações afetivas";

Hipótese 4: "Quanto maior o nível de conhecimento prévio objetivo do consumidor, menor o nível de dificuldade percebido na tarefa";

Hipótese 5: "Quanto maior o nível de conhecimento prévio subjetivo, menor o nível de dificuldade percebido na tarefa";

Hipótese 6: "Quanto maior a experiência anterior de uso do produto, maior o conhecimento prévio objetivo";

Hipótese 7: "Quanto maior a experiência anterior de uso do produto, maior o conhecimento prévio subjetivo";

Hipótese 8: "Quanto maior a importância de informações instrumentais, mais provável a busca de informações de fonte fraca"; e 
Hipótese 9: "Quanto maior o nível de conhecimento prévio subjetivo, maior o uso de informações instrumentais".

Coeficientes de correlação entre os construtos foram estimados por DUHAN et al. em um contexto de busca de informação sobre serviços médicos de obstetrícia (185 mulheres em idade reprodutiva foram entrevistadas). Os resultados estão relacionados na coluna IV da Tabela 1; infelizmente, o nível de significância das correlações não foi relatado, mas é razoável admitir que se aproximam da significância dos efeitos principais padronizados estimados por equações estruturais, reproduzidos na coluna III.

Os resultados obtidos por DUHAN et al. (1997) são coerentes com a proposição geral de BROWN e REINGEN (1987) de que existem diversas influências atuando sobre a escolha que consumidores fazem dos tipos de fontes de recomendação. Especificamente, DUHAN et al. (1997) concluíram que há duas "trilhas" causais distintas: a escolha de fontes fortes é influenciada pela dificuldade da tarefa e pelo conhecimento prévio subjetivo e objetivo do consumidor; e a escolha de fontes fracas é influenciada pela importância atribuída às informações instrumentais e pelo conhecimento prévio subjetivo do consumidor.

\section{Fontes de Informação na Compra de Automóveis Novos}

O segmento de bens duráveis oferece condições favoráveis ao estudo do comportamento do consumidor na etapa de busca de informação, pois categorias de produtos de maior valor de aquisição tomam mais tempo e são objetos de análises mais cuidadosas dos consumidores (HOYER e BROWN, 1990 e RUNYON, 1977). Os consumidores fazem menos esforço cognitivo para escolher pasta de amendoim do que para escolher um par de tênis ou ainda um automóvel. Assim, decidimos aplicar o modelo de DUHAN et al. (1997) ao processo de escolha de um veículo novo (Tabela 2).

PIRES e MARCHETTI (2000) seguiram justamente esta estratégia, isto é, a aplicação do modelo de DUHAN et al. (1997) na análise dos fatores que influenciam a escolha da fonte de informações por parte de compradores de automóveis novos. No entanto, os dados coletados por PIRES e MARCHETTI a respeito de 128 compradores da cidade de Maringá, norte do estado do Paraná, confirmam, ao nível de significância de 5\%, apenas as relações existentes entre os construtos de experiência prévia e conhecimento prévio subjetivo; estes autores interpretam que as demais associações postuladas no modelo original de DUHAN et al. não apresentam significância no contexto de seu trabalho. Parece-nos relevante, porém, que as hipóteses H1, H2 e H8 sejam significativas ao nível de $10 \%$. 


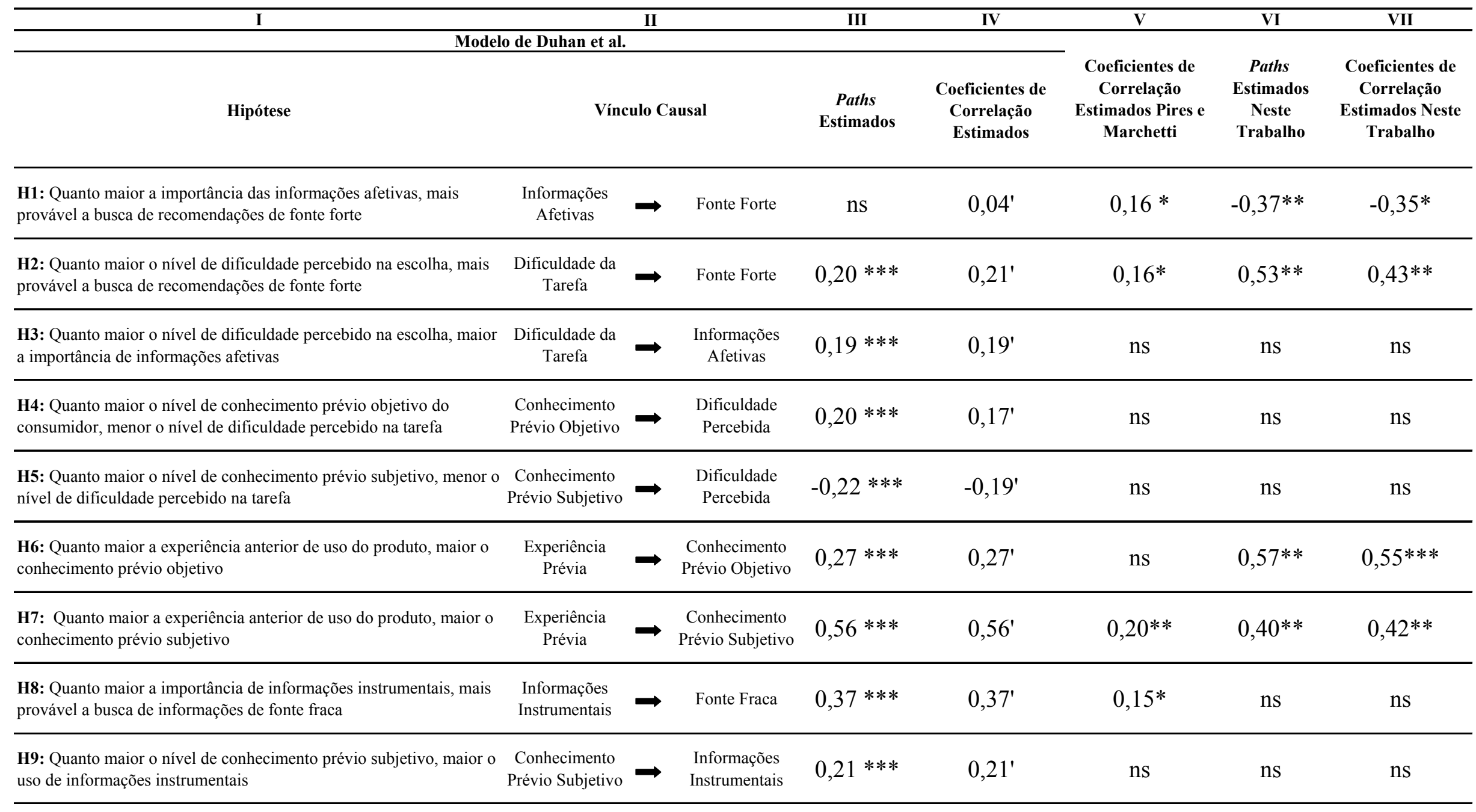

* Significativo a $10 \% \quad * *$ Significativo a $5 \% \quad * * *$ Significativo a $1 \%$ ' Significância não reportada

Tabela 1: Sistematização dos Trabalhos Comparados Neste Artigo 


\begin{tabular}{|l|l|l|l|l|}
\hline & DUHAN et. al. & $\begin{array}{l}\text { PIRES } \\
\text { MARCHETTI }\end{array}$ & $\begin{array}{l}\text { PONCHIO et. al. } \\
\text { (este estudo) }\end{array}$ \\
\hline Contexto & $\begin{array}{l}\text { Escolha de serviços } \\
\text { médicos de obstetrícia }\end{array}$ & $\begin{array}{l}\text { Compra recente de } \\
\text { veículos novos }\end{array}$ & $\begin{array}{l}\text { Escolha de automóvel } \\
\text { novo }\end{array}$ \\
\hline $\begin{array}{l}\text { Método de Coleta de } \\
\text { Dados }\end{array}$ & Cenário Questionário & $\begin{array}{l}\text { Questionário pós- } \\
\text { compra }\end{array}$ & $\begin{array}{l}\text { Cenário Questionário } \\
\text { Experimento }\end{array}$ \\
\hline Amostra & $\begin{array}{l}185 \text { de } 800 \\
\text { questionários } \\
\text { (mulheres em idade } \\
\text { reprodutiva) }\end{array}$ & $\begin{array}{l}128 \text { questionários } \\
\text { (compradores de } \\
\text { veículos novos) }\end{array}$ & $\begin{array}{l}122 \text { de } \\
\text { convidados (turmas } \\
\text { da FGVIEAESP) }\end{array}$ \\
\hline
\end{tabular}

Tabela 2 - Resumo do Contexto dos Estudos Comparados

Por outro lado, correlações não postuladas mostraram-se fortes e significativas. Diante destes resultados, PIRES e MARCHETTI (2000) recomendaram duas linhas de conduta: realização de novas aplicações do modelo, na forma original proposta por DUHAN et al, a contextos diferentes; e reformulação, incremental ou radical, do modelo original.

Neste trabalho, optamos pela primeira estratégia e fazemos uma aplicação, em contexto novo, do modelo de DUHAN et al.(1997) tal como originalmente proposto. Buscamos, com isso, coletar mais evidências sobre seu ajuste ou desajuste. Foi mantido o foco no processo de escolha de veículos novos adotado por PIRES e MARCHETTI (2000). Por outro lado, tendo em vista que a implementação das medidas de construtos teóricos pode ser a origem de problemas capazes de impossibilitar a comprovação de um modelo (PEDHAZUR e SCHMELKIN, 1991), optamos por utilizar novos instrumentos de coleta e uma estratégia mista: experimento complementado por questionário.

\section{Um Experimento pela Internet}

A idéia de utilizar um experimento computadorizado para observar o consumidor não é nova. JOHNSON et al. (1997) desenvolveram um método de coleta de dados - batizado por eles de Process-Assisted Choice Modeling (PACMod) - cujo ponto de partida é monitorar a forma como as informações de que os consumidores dispõem são seqüencialmente coletadas, editadas e avaliadas. O programa de computador disponibiliza ao participante do experimento informações a respeito de produtos e armazena, em um banco de dados, quais das informações disponíveis foram acessadas e por quanto tempo elas foram visualizadas.

Em nosso estudo, a técnica de PACMod foi combinada ao uso de questionários. Os consumidores foram convidados a acessar um site na Internet, em que deveriam responder a duas séries de perguntas e tomar uma decisão.

\section{Descrição do site}

O site criado para o experimento (Figura 2) é composto por:

Uma tela de boas-vindas apresentando a pesquisa.

Um questionário inicial, de 10 questões, por meio do qual foram coletados dados demográficos (sexo e idade), de experiência prévia e de conhecimento prévio, objetivo e subjetivo, sobre automóveis. 
Uma seqüência de telas que formam o experimento, e um questionário final, em que se apura a decisão do participante quanto à escolha do produto e a forma como o participante avaliou itens de informação instrumentais e afetivos.

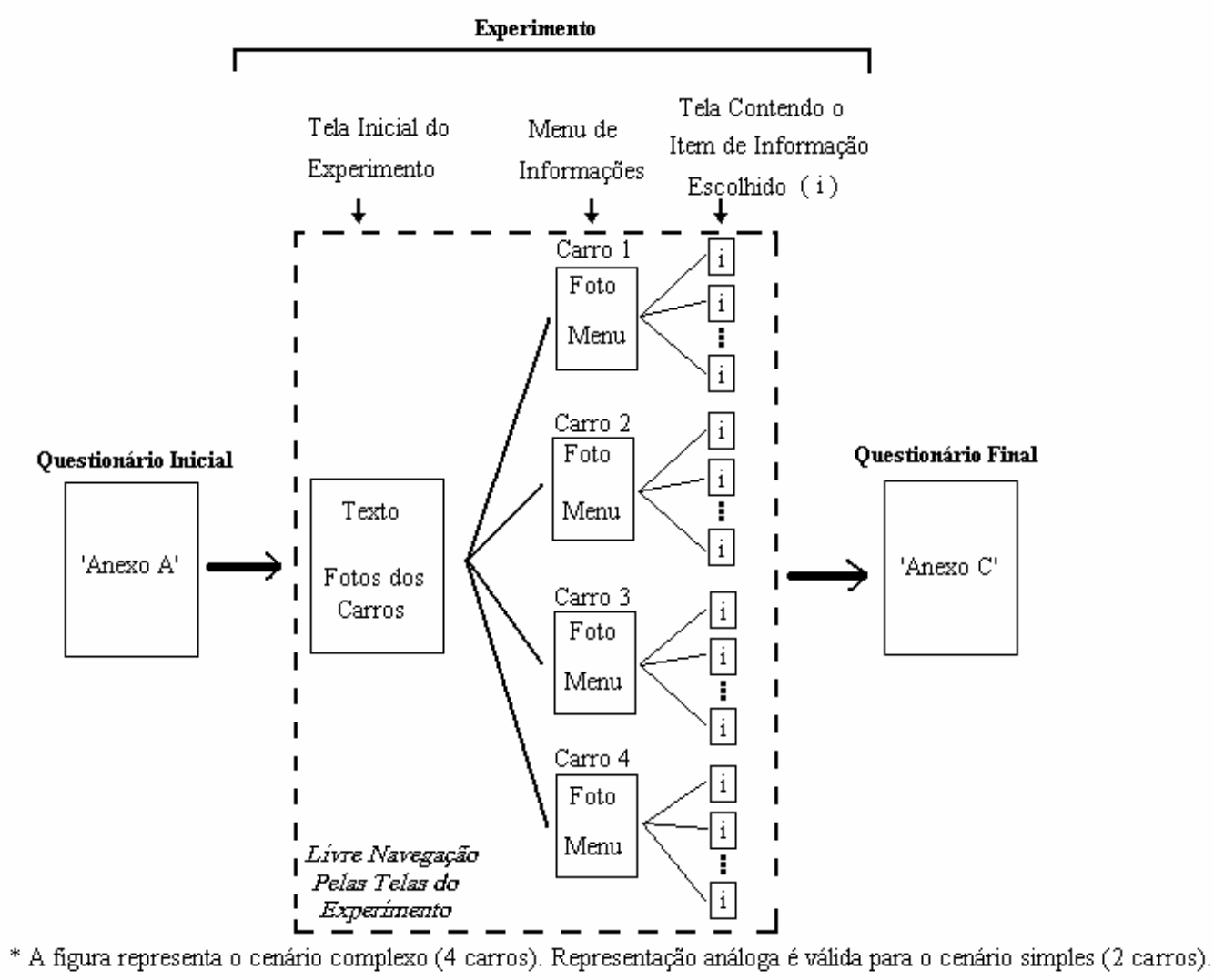

Figura 2 - Esquema do Site Desenvolvido para a Realização Deste Projeto de Pesquisa

\section{O Experimento}

O experimento consiste em pedir ao participante na pesquisa que se imagine vencedor de um concurso promovido por uma emissora de televisão. Como prêmio, o participante/vencedor deve escolher um automóvel dentre aqueles disponibilizados no site.

Para metade dos participantes são apresentados apenas dois veículos (Chevrolet Vectra GLS 2.2 e Honda Civic EX 1.7); para a outra metade, são oferecidos quatro veículos (os dois anteriores, mais o Toyota Corolla SE-G 1.8 e o Fiat Marea HLX 2.4). Todos os veículos são do tipo sedã médio e de produção nacional. Possuem valores de mercado semelhantes e competem pelo mesmo mercado.

Por meio da variação do número de alternativas a serem examinadas pelo consumidor, pretendeu-se controlar a complexidade da tarefa proposta.

O servidor que hospeda o site foi programado para que as duas versões de cenário sejam disponibilizadas de forma alternada. Assim, os participantes na pesquisa cujo número de acesso ao site for ímpar deparam-se com o cenário em que devem escolher um entre apenas dois automóveis; e os participantes cujo número de acesso ao site for par deparam-se com quatro carros, dentre os quais devem escolher um. 
Mateus Canniatti Ponchio - Francisco José Epósito Aranha Filho - André Luiz Silva Samartini

Uma vez conhecidas as alternativas, o participante pode navegar pelo site em busca de informações sobre os veículos. Embora as opções de escolha variassem entre duas e quatro, os tipos de informações disponíveis, bem como os questionários, eram semelhantes.

\section{Dinâmica da navegação}

Após o participante enviar o primeiro questionário, tem início o experimento, cuja tela inicial consiste de um breve texto introdutório seguido por fotos dos carros disponíveis para a escolha (Figura 3). Para cada carro são disponibilizados diversos itens de informação, agrupados em menus dinâmicos segundo a fonte de recomendação (Figura 4).

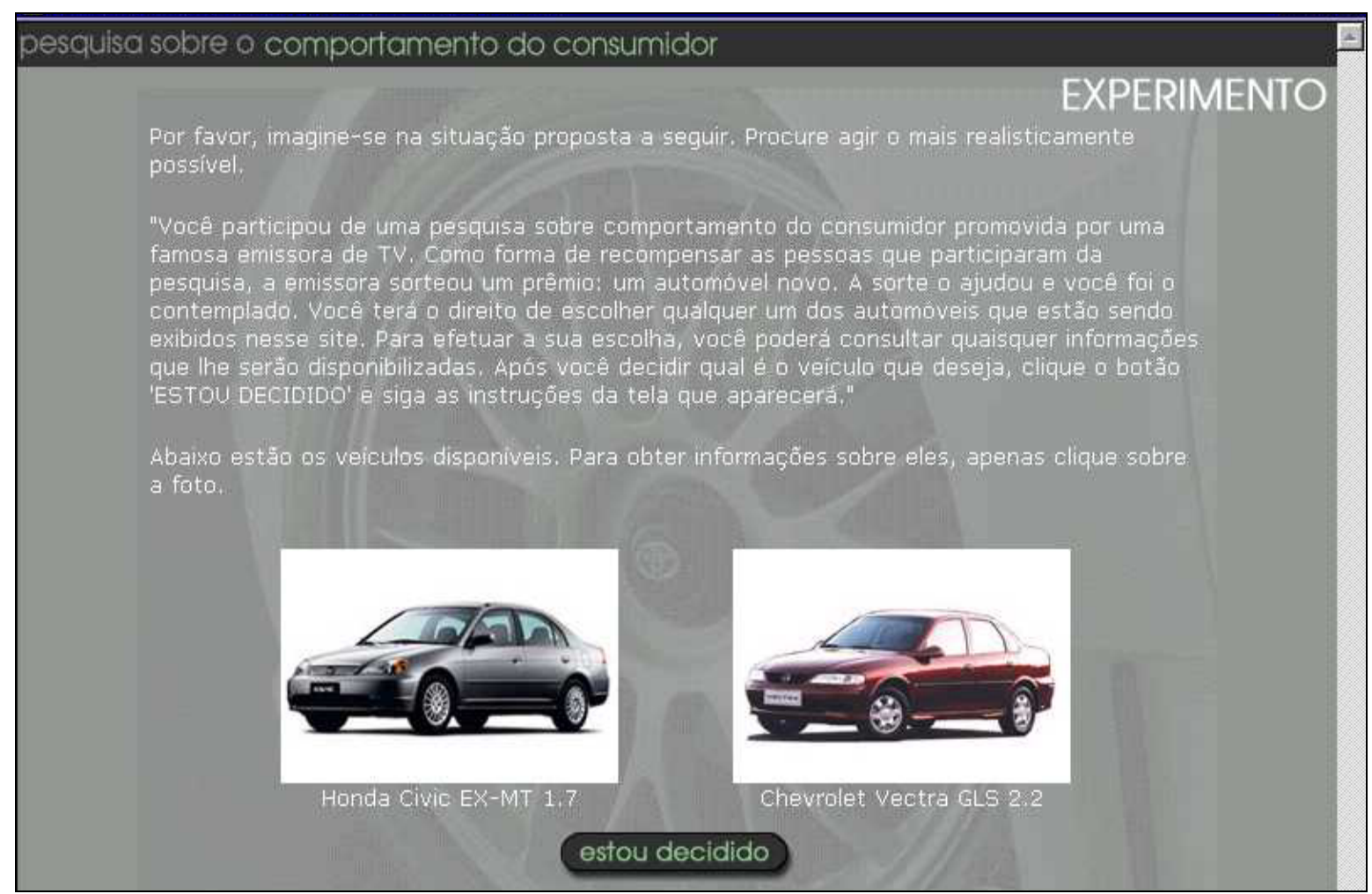

Figura 3 - Tela de Apresentação do Experimento 


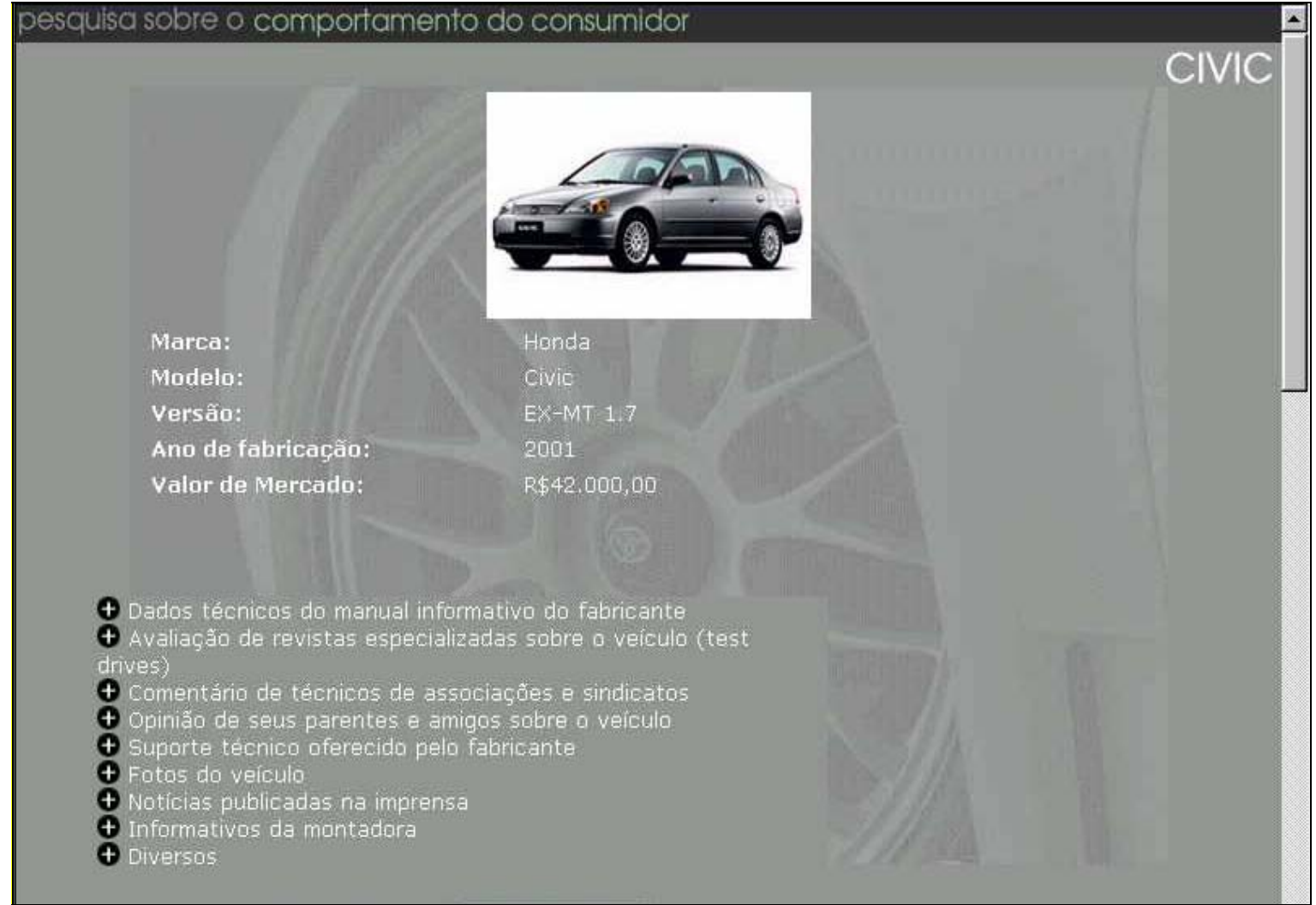

Figura 4 - Menu Dinâmico de Informações Sobre Veículo

No total, há 70 itens de informação disponíveis no cenário simples e 140 no cenário complexo. Não há nenhum tipo de restrição quanto à quantidade de itens de informação passíveis de serem consultados, ficando essa decisão a critério do participante. $\mathrm{O}$ número de consultas por participante está representado na Figura 5; da distribuição da variável se depreende que, conforme esperado, as informações disponíveis não são esgotadas pelos participantes do experimento. Estes, a qualquer momento, podem clicar no botão "Estou decidido". Assim procedendo, os participantes são levados à tela final da pesquisa, composta pelo segundo questionário.

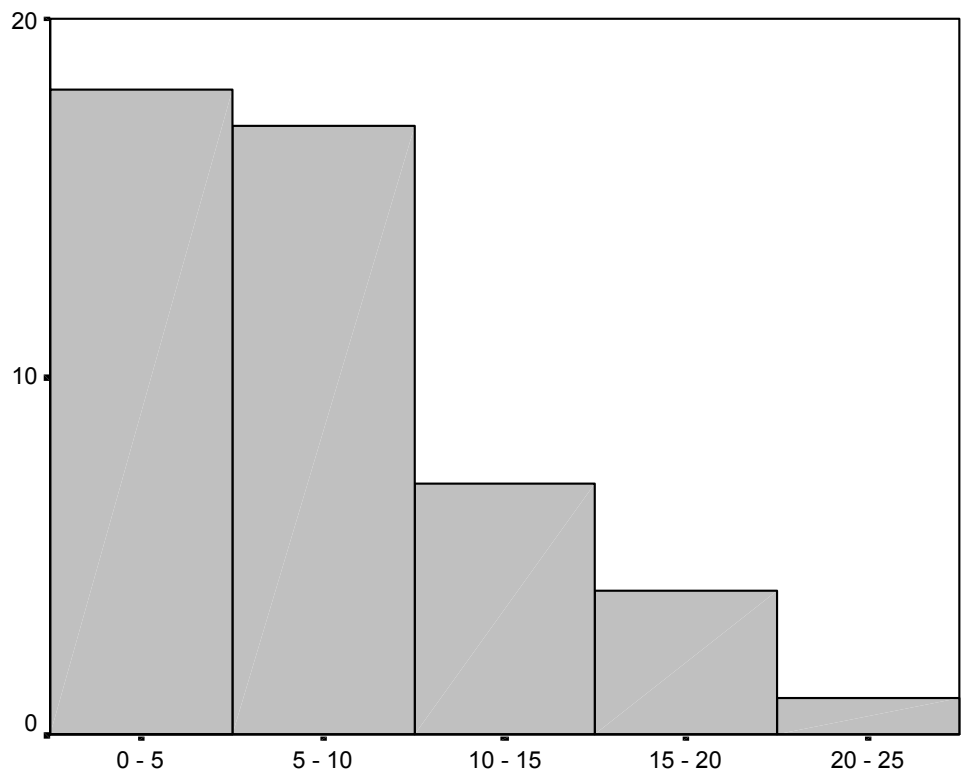

Figura 5 - Distribuição do Total de Itens de Informação Consultados por Participante

Os dados referentes às variáveis número de fontes fortes e número de fontes fracas consultadas foram retirados da navegação dos participantes pelo site. 
Em resumo, o banco de dados do site da pesquisa armazenou: respostas aos questionários; páginas acessadas pelo participante (consequentemente, itens de informação consultados e respectivas fontes); e o tempo gasto pelo participante em cada uma das páginas.

\section{Coleta de Dados}

A coleta de dados foi realizada em três semanas de março de 2001. Durante esse período, o site foi disponibilizado na Internet. Foram distribuídos 500 convites de participação a alunos de graduação, mestrado e doutorado da Escola de Administração de Empresas de São Paulo da Fundação Getulio Vargas, em turmas escolhidas por conveniência.

$\mathrm{Na}$ data do fechamento da coleta havia 136 questionários respondidos, dos quais $122 \mathrm{em}$ condições de serem aproveitados. Nada se pode afirmar sobre o percentual de respostas obtidas dentre os 500 convidados, pois o único pré-requisito para participar do experimento era possuir o endereço do site na Internet. Dessa forma, não houve controle sobre pessoas que não receberam o convite diretamente, mas foram encaminhadas por outros participantes. Apenas 3,7\% dos respondentes, no entanto, não eram alunos.

\section{Metodologia de Análise}

O ajuste do modelo apresentado na Figura 1 aos dados coletados no experimento realizado no site desta pesquisa foi obtido e testado por meio do uso do software LISREL 8.30 (JORESKOG e SORBOM, 1999). Equações estruturais foram escolhidas como técnica de análise em decorrência da natureza das relações a serem testadas, do tipo caminhos causais (MARUYAMA, 1997; HAIR et al, 1995, URDAN e RODRIGUES, 1998), e principalmente para permitir a comparação dos resultados com os de DUHAN et al.(1997). Embora a amostra utilizada seja pequena no contexto de equações estruturais, ela foi suficiente para a estimação do modelo simples a que nos restringimos.

A comparação com os resultados de PIRES e MARCHETTI (2000) foi feita apenas com base na matriz de correlações, uma vez que estes autores não utilizaram equações estruturais.

Assim, são comparáveis as colunas III e VI da Figura 1, relativas a caminhos causais (paths) estimados respectivamente por DUHAN et al. (1997) e por nós, no presente artigo. E as colunas IV, V e VII, relativas a correlações, e estimadas respectivamente por DUHAN et al. (1997), PIRES e MARCHETTI (2000), e por nós, neste artigo.

\section{Resultados}

A coluna VI da Tabela 1 apresenta os caminhos causais estimados neste trabalho. Ao nível de significância de 5\%, mostraram-se significativas apenas a Hipóteses 1 e 2, ou seja, o número de fontes fortes consultadas depende respectivamente da importância atribuída a informações afetivas e da dificuldade da tarefa e as Hipóteses 6 e 7, ou seja, o conhecimento respectivamente objetivo $e$ subjetivo dependem da experiência no uso do produto.

Assim como aconteceu com DUHAN (1997), não há evidência contrária ao ajuste do modelo, pois o nível descritivo para o teste de ajuste íntimo obtido foi de 0,17 . Como se sabe, no entanto, a falta de evidência para rejeição do modelo não significa que ele seja verdadeiro.

E, assim como aconteceu com PIRES e MARCHETTI (2000), a correlação entre fontes fortes e fracas mostrou-se relativamente alta e significativa (coeficiente de correlação de $0,42 * *$ ). 


\section{Discussão}

Embora os coeficientes estimados para as hipóteses $\mathrm{H} 1$ e $\mathrm{H} 2$ tenham se mostrado significativos a 5\%, o sinal da correlação obtida para $\mathrm{H} 1$ contraria a postulação do modelo, pois a relação encontrada entre informações afetivas e fontes fortes foi inversa à esperada.

Talvez a hipótese H1 seja uma relação instável, ou muito sensível a pequenas variações de contexto, como tipo de produto ou tipo de público-alvo. No estudo de DUHAN et al. não foi encontrado vínculo causal entre as variáveis 'informações afetivas' e 'fontes fortes', e, em PIRES e MARCHETTI, o valor encontrado para a correlação entre essas variáveis foi de 0,16 , significativa a $10 \%$. Alternativamente, pode-se imaginar que a valorização de informações afetivas não implique na busca ativa dessas informações. É possível que elas sejam coletadas amplamente, e de maneira passiva, antes da situação de compra, ou da situação experimental. De qualquer forma, existe uma relação indireta entre informações afetivas e fontes fortes, já que ambas sofrem influência da variável complexidade da tarefa.

Quanto às hipóteses H2, H6, H7 e H8, há coincidência de conclusões entre dois ou três dos estudos (Figura 4).

A experiência prévia no uso do produto influi de maneira positiva e significativa sobre o grau de conhecimento prévio objetivo (H6) e subjetivo (H7). Ou seja, a experiência anterior contribui para que o consumidor aprenda sobre o produto e tenha consciência do seu conhecimento.

Verifica-se também que a correlação entre o grau de complexidade da tarefa e a preferência por fontes de recomendações fortes mostrou-se positiva e significativa (H2). Este resultado converge com o trabalho de BROWN e REINGEN (1987), cujos resultados associam um aumento da dificuldade da escolha a uma menor confiança do consumidor em si mesmo. Para compensar este déficit, o consumidor busca apoio em fontes similares a si, tais como amigos e parentes. No limite, os consumidores, frente a situações muito complexas, delegariam a escolha para alguém em que eles confiassem.

As hipóteses H3, H4, H5 e H9, entretanto, somente foram confirmadas pelo estudo de DUHAN et al. (1997). Este parece ser um ponto de fragilidade no modelo, pois sugere que pode haver diferença nas relações de causalidade para diferentes categorias de produto. Além disso, duas novas hipóteses podem ser formuladas:

A quantidade de conhecimento anterior não influencia o padrão de busca de informações (o que parece pouco razoável);

Existem diferentes perfis de consumidores; as correlações entre os construtos de interesse são fortes e significativas dentro do perfil, porém contrárias entre perfis, anulando-se quando estes são analisados em conjunto. Em outras palavras, sugerimos que a reunião de padrões de grupos distintos de consumidores acaba ocultando as diferenças entre os segmentos, e que isso não aconteceria se os segmentos fossem considerados em separado. Em um contexto de distribuição geográfica de idosos, o ocultamento de um fenômeno pela união de segmentos com comportamento distinto foi identificado por ARANHA (1999). Na mesma linha, estudando os benefícios que compradores de automóveis novos podem obter com a utilização da Internet como fonte de informação, HERNANDEZ (2001) mostra que a identificação e comprovação do efeito da consulta à Internet sobre a obtenção de descontos na aquisição de veículos novos depende da análise dos segmentos em separado ou em conjunto. 
Grau de conhecimento prévio do consumidor
Variáveis de ligação
Tipo de fonte de informação

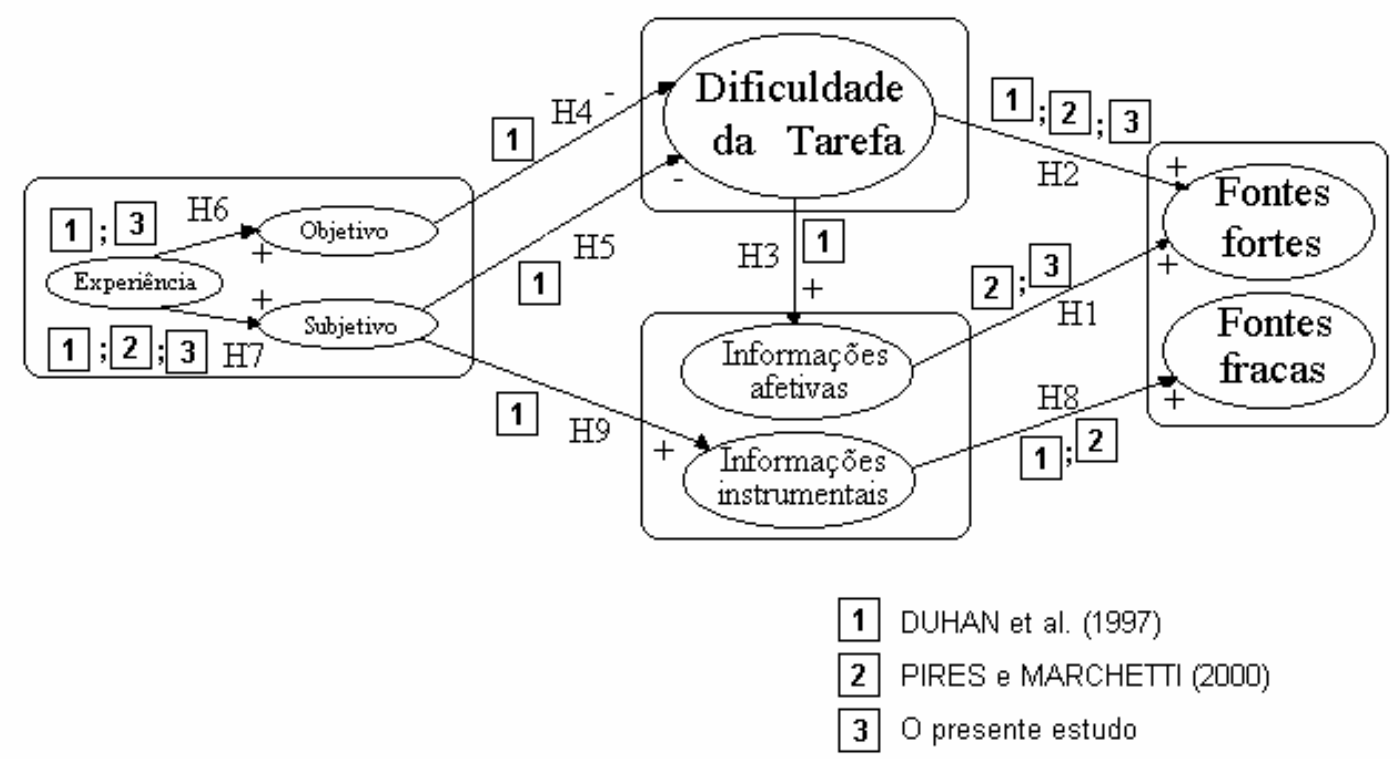

Figura 6 - Fragilidade das Hipóteses H3, H4, H5 e H9

Finalmente, a correlação entre os dois tipos de fontes, não postulada pelo modelo mas constatada nesta pesquisa, talvez esteja sinalizando a existência de uma nova dimensão - o grau de atividade ou empenho do consumidor.

Este grau de atividade representa a disposição dos consumidores em investir esforços na etapa de busca: consumidores com alto empenho dedicariam-se diligentemente à tarefa, acionando os dois tipos de fontes de informação; ao passo que consumidores com baixo empenho, mesmo diante da necessidade, buscariam pouca informação, e de qualquer fonte. É concebível que este efeito predomine sobre a diferença de composição da busca (mix de fontes fortes e fracas) realizada pelos consumidores.

No artigo já citado, HERNANDEZ (2001) constatou que compradores de automóveis novos que consultaram a Internet investiram, em média, 20,7 horas em procura por informações, das quais 3,8 horas consultando a Internet e 16,9 horas procurando em outras fontes de informação; enquanto compradores que não consultaram a Internet investiram, em média, 14,4 horas em procura. Portanto, a diferença entre o tempo investido em procura pelos dois grupos não se deveu exclusivamente ao tempo adicional utilizado na Internet: o usuário de Internet também aplicou mais tempo à procura em outras fontes, mostrando-se mais ativo que o não usuário.

Mesmo que a correlação do nível de acesso aos dois tipos de fontes tenha sido exacerbada em decorrência de uma curiosidade despertada pelo próprio experimento, com o desejo de conhecer o site sendo interpretado como busca mais ativa, deve-se considerar que a correlação já havia sido identificada em PIRES e MARCHETTI (2000). Ali não pode ser atribuída a um artefato da técnica experimental. 


\section{Limitações, Conclusões e Desdobramentos}

Os resultados relatados neste artigo devem ser usados com cuidado, já que estão baseados em amostra de conveniência, com número relativamente pequeno de respostas, algumas das quais, incompletas.

A tecnologia empregada no site deve ser aperfeiçoada visto que, durante o período de coleta de dados, o servidor ficou 'fora do ar' em algumas ocasiões. Além disso, as figuras existentes no site fazem a navegação lenta se a velocidade e a qualidade da conexão do usuário não forem altas; isso pode desincentivá-lo a conduzir o experimento até o final. Sem esses inconvenientes, o número de respondentes da pesquisa poderia ter sido maior.

Como se sabe, conclusões obtidas em ambiente experimental podem não ser diretamente traduzíveis para situações reais. $\mathrm{O}$ argumento de que experimentos podem induzir comportamentos diferentes do que aconteceriam em situações não controladas, no entanto, é mitigado em razão das semelhanças obtidas em estudos realizados em contextos diferentes.

Este trabalho contribui com estudos anteriores na área de tomada de decisão com base em recomendações, ampliando as linhas de pesquisa através da investigação das influências na escolha de uma pessoa por fontes de recomendações. O modelo testado originalmente propõe que o grau de conhecimento anterior, o nível de dificuldade percebido e os tipos de informações procurados pelo consumidor influenciam sua escolha por fontes de recomendações. Após o confrontamento dos três estudos apresentados, entretanto, concluímos que, se por um lado os construtos "nível de dificuldade" e "tipos de informações" mostram expressiva ligação com as fontes de recomendações escolhidas, por outro, as relações envolvendo o construto "grau de conhecimento anterior" mostraram-se significativas apenas no primeiro dos estudos. Esses dados nos levam a crer que categorias de produtos e diferenças de contextos são fatores que influenciam a escolha dos consumidores por fontes de recomendações, inclusive possivelmente dominando os fatores controlados em nosso experimento. Novos estudos são necessários para investigar estas questões.

De qualquer forma, os resultados obtidos em parte confirmam o modelo de DUHAN et al, e em parte sugerem novas possibilidades para aperfeiçoamento do modelo: segmentação dos usuários por perfil psicológico e incorporação do grau de empenho do consumidor na busca de informações. Levanta, ainda, a possibilidade de independência entre o conhecimento anterior do produto e a preferência por fontes de informação fortes e fracas.

\section{Artigo recebido em 19/10/2001. Aprovado em 21/05/2002.}




\section{BIBLIOGRAFIA}

AHTOLA, Olli T. The Vector Model of Preferences: An Alternative to the Fishbein Model. Journal of Marketing Research, Chicago, v. 12, p. 52-59, Feb. 1975.

ARANHA, Francisco. Síndrome do Óculos para Perto. InfoGEO, Curitiba, v.2, n. 7, p. 31-2, 1999.

BEATTY, Sharon E., SMITH, Scott M. External Search Effort: An Investigation Across Several Product Categories. Journal of Consumer Research, Gainesville, v. 14, p. 83-95, June 1987.

BEN-SIRA, Zeev. The Function of the Professional's Affective Behavior in Client Satisfaction: A Revised Approach to Social Interaction Theory. Journal of Health and Social Behavior, v. 17, p. 3-11, Mar. 1976.

BEN-SIRA, Zeev. Affective and Instrumental Components in the Physician - Patient Relationship: An Additional Dimension of Interaction Theory. Journal of Health and Social Behavior, v. 21, p. 170-180, June 1980.

BETTMAN, James R., PARK, Whan C. Effects of Prior Knowledge and Experience and Phase of the Choice Process on Consumer Decision Processes: A Protocol Analysis. Journal of Consumer Research, Gainesville, v. 7, p. 234-248, Dec. 1980.

BETTMAN, James R., ZINS, Michel A. Information Format and Choice Task Effects in Decision Making. Journal of Consumer Research, Gainesville, v. 6, p. 141-153, Sept. 1979.

BRONIARCZYK, Susan M., HOYER, Wayne D., McALISTER, Leigh. Consumers' Perceptions of the Assortment Offered in a Grocery Category: The Impact of Item Reduction. Journal of Marketing Research, Chicago, v. 35, p. 166-176, May 1998.

BROWN, Jacqueline J., REINGEN, Peter H. Social Ties and Word-of-Mouth Referral Behavior. Journal of Consumer Research, Gainesville, v. 14, p. 350-362, Dec. 1987.

BRUCKS, Merrie. The Effects of Product Class Knowledge on Information Search Behavior. Journal of Consumer Research, Gainesville, v. 12, p. 1-16, June 1985.

BRUCKS, Merrie, MAC INNIS, Deborah J. Advances in Consumer Research. Provo: Association for Consumer Research, 1997, 560p.

COUPEY, Eloïse, IRWIN, Julie F., PAYNE, John W. Product Category Familiarity and Preference Construction. Journal of Consumer Research, Gainesville, v. 24, p. 459-468, Mar. 1998.

DUHAN, Dale F., JOHNSON, Scott D., WILCOX, James B., HARRELL, Gilbert D. Influences on Consumer Use of Word-of-Mouth Recommendation Sources. Journal of the Academy of Marketing Science, Coral Gables, v. 25, n. 4, p. 283-295, Fall 1997.

HAIR, Joseph, ANDERSON, Rolph, TATHAM, Ronald, BLACK, Willian. Multivariate Data Analysis. 4a. ed. Englewood Cliffs: Prentice Hall, 1995, 745p.

HERNANDEZ, José Mauro da Costa. Seria a Internet um Atalho Para Descontos Maiores na Compra do Automóvel Novo? Uma Investigação Empírica Sobre os Benefícios da Procura. In: 
Encontro Anual da Associação Nacional de Pós-Graduação em Administração, 25, (2001), Campinas, SP. Resumo dos Trabalhos ENANPAD 2001. Rio de Janeiro: ANPAD, 367p.

HOYER, Wayne D. An Examination of Consumer Decision Making for a Common Repeat Purchase Product. Journal of Consumer Research, Gainesville, v. 11, p. 822-829, Dec. 1984.

HOYER, Wayne D., BROWN, Steven P. Effects of Brand Awareness on Choice for a Common, Repeat-Purchase Product. Journal of Consumer Research, Gainesville, v. 17, p. 141-147, Sept. 1990.

JOHNSON, Eric J., MEYER, Robert J., HARDIE, Bruce G., ANDERSON, Paul. Watching Consumers Decide. Journal of Marketing Research, Chicago, v. 9, p. 32-37, Winter 1997.

JORESKOG, Karl, SORBOM, Dag. LISREL 8.30. Chicago: Scientific Software International, Inc, 1999. Software.

KIM, Chankon, KHOURY, Majeed. Task Complexity and Contingent Information Processing in the Case of Couple's Decision Making. Journal of the Academy of Marketing Science, Coral Gables, v. 25, p. 32-43, Fall 1987.

KOTLER, Philip. Administração de Marketing: Análise , Planejamento, Implementação e Controle. 5a ed. São Paulo: Atlas, 1998, 693p.

LUSSIER, Denis A., OLSHAVSKY, Richard W. Task Complexity and Contingent Processing in Brand Choice. Journal of Consumer Research, Gainesville, v. 6, p. 154-165, Sept. 1979.

MARUYAMA, Geoffrey. Basics of Structural Equation Modeling. Newburry Park: Sage, 1997.

MEYER, Robert J., A Model of Multiattribute Judgments Under Attribute Uncertainty and Informational Constraint. Journal of Marketing Research, Chicago, v. 18, p. 428-441, Nov. 1981.

MIDGLEY, David F. Patterns of Interpersonal Information Seeking for the Purchase of a Symbolic Product. Journal of Marketing Research, Chicago, v. 20, p. 74-83, Feb. 1983.

NEWELL, A., SIMON, H. Human Problem Solving. Englewood Cliffs: Prentice Hall, 1972, 920p.

NEWMAN, Joseph W., STAELIN, Richard. Prepurchase Information Seeking for New Cars and Major Household Appliances. Journal of Marketing Research, Chicago, v. 9, p. 249-257, Aug. 1972.

NEWMAN, Joseph W., STAELIN, Richard. Information Sources of Durable Goods. Journal of Advertising Research, New York, v. 13, p. 19-29, Apr. 1973.

OLSHAVSKY, Richard W., GRANBOIS, Donald H. Consumer Decision Making - Fact or Fiction? Journal of Consumer Research, Gainesville, v. 6, p. 93-100, Sept. 1979.

PAQUETTE, Laurence, KIDA, Thomas. The Effect of Decision Strategy and Task Complexity on Decision Performance. Organizational Behavior and Human Decision Processes, Orlando, v. 41, p. 128-142, 1988. 
PAYNE, John W. Task Complexity and Contingent Processing in Decision Making: An Information Search and Protocol Analysis. Organizational Behavior and Human Performance, v. 16, p. 366-387, 1976.

PEDHAZUR, Elazar, SCHMELKIN, Liora P. Measurement, Design and Analysis: An Integrated Approach. Hillsdale: Lawrence Erlbaum Associates, 1991, 819p.

PIRES, Vicente C., MARCHETTI, Renato Z. Fatores Influenciadores na Escolha da Fonte de Informação na Compra de Automóveis Novos: A importância da comunicação boca-a-boca. In: Encontro Anual da Associação Nacional de Pós-Graduação em Administração, 24, (2000), Florianópolis, SC. Resumo dos Trabalhos ENANPAD 2000. Rio de Janeiro: ANPAD, 2000. 304p.

PUNJ, Girish N., STAELIN, Richard. A Model of Consumer Information Search Behavior for New Automobiles. Journal of Consumer Research, Gainesville, v. 9, p. 366-380, Mar. 1983.

PUTSIS JR., William P., SRINIVASAN, Narasimhan. Buying or Just Browsing? The Duration of Purchase Deliberation. Journal of Marketing Research, Chicago, v. 31, p. 393-402, Aug. 1994.

SHELUGA, David A., JACCARD, James, JACOBY, Jacob. Preference, Search and Choice: An Integrative Approach. Journal of Consumer Research, Gainesville, v. 6, p. 166-176, Sept. 1979.

SPÍNOLA, Francisco Alcântara. Auto-Conceito dos Compradores Norte-Americanos e Brasileiros e Suas Estruturas de Decisão: Pesquisa Internacional Comparada. São Paulo: USP/Faculdade de Economia, Administração e Contabilidade, 1995. 167p. (Dissertação, Mestrado, Marketing).

URDAN, André T., RODRIGUES, Arnaldo R. O Modelo do Índice de Satisfação do Cliente NorteAmericano: Um Exame Inicial no Brasil, com Equações Estruturais. In: Encontro Anual da Associação Nacional de Pós-Graduação em Administração, 22, (1998), Foz do Iguaçu, PR. Resumo dos Trabalhos ENANPAD 1998. Rio de Janeiro: ANPAD, 221p.

WELLS, William D, LO SCIUTO, Leonard A. Direct Observation of Purchasing Behavior. Journal of Marketing Research, Chicago, v. 3, p. 227-233, Aug. 1966.

WRIGHT, Peter. Consumer Choice Strategies: Simplifying Vs. Optimizing. Journal of Marketing Research, Chicago, v. 12, p. 60-67, Feb. 1975. 